\title{
Design of Fully Digital Controlled Shaped Beam Synthesis Using Differential Evolution Algorithm
}

\author{
D. Mandal, ${ }^{1}$ A. Chatterjee, ${ }^{2}$ and A. K. Bhattacharjee ${ }^{3}$ \\ ${ }^{1}$ Department of Electronics and Communication Engineering, Bengal College of Engineering and Technology, \\ West-Bengal, Durgapur-713212, India \\ ${ }^{2}$ Department of Electronics and Communication Engineering, Dr. B. C. Roy Engineering College, West-Bengal, Durgapur-713206, India \\ ${ }^{3}$ Department of Electronics and Communication Engineering, National Institute of Technology Durgapur, West-Bengal, \\ Durgapur-713209, India
}

Correspondence should be addressed to D. Mandal; deb.mandal22@gmail.com

Received 20 August 2013; Revised 3 October 2013; Accepted 3 October 2013

Academic Editor: Dau-Chyrh Chang

Copyright (c) 2013 D. Mandal et al. This is an open access article distributed under the Creative Commons Attribution License, which permits unrestricted use, distribution, and reproduction in any medium, provided the original work is properly cited.

\begin{abstract}
A shaped beam synthesis from a concentric ring array has been presented. Two different cases are considered. In the first case, a flat-top beam pattern and, in the second case, a $\operatorname{cosec}^{2}$ beam pattern have been generated. In both the cases, it has been ensured that the obtained beam patterns are not restricted in any single predefined $\varphi$-cut of the patterns; rather, their characteristics are retaining within a range of predefined $\varphi$-planes with some minor variations. The desired beam pattern under each of the individual case is obtained by finding optimum discrete excitations of the array elements. The optimum 4-bit amplitudes generated by four-bit digital attenuators and 5-bit phases generated by 5-bit digital phase shifters are computed using Differential Evolution (DE) Algorithm. To illustrate the effectiveness of DE, the two beam patterns with specified characteristics are computed from the same array using Particle Swarm Optimization (PSO) algorithm and Genetic algorithm (GA) by finding out optimum discrete excitations among the elements. Results clearly show the superiority of DE over PSO and GA to handle the presented problem.
\end{abstract}

\section{Introduction}

In mobile, satellite, and radar communication the important parameters are signal quality, system coverage, spectral efficiency, and so forth. To achieve these, efficient antenna design is the primary requirement. Antenna array synthesis is required to find radiation patterns from different array geometry and make these obtain patterns closer to their desired patterns either by varying its elements amplitude and phase or by reconfiguration of the array geometry. In various applications shaped beams are often required but major problems faced by shaped beams are high side lobe level and ripple. To minimize high sidelobe and ripple, an efficient evolutionary optimization algorithm has been chosen which is able to find out 4-bit optimum discrete elements amplitude and 5-bit optimum discrete phases of the array elements to achieve the desired shaped beam. Several approaches reported in the literature for generating shaped beams [1-6] are as follows.
Azevedo proposed a technique based on FFT to generate shaped beams of cosec and flat-top pattern from a linear array antenna through the control of nonuniformly samples of the array factor, both in amplitude and phase [3]. Lei et al. generate low sidelobe cosecant square-shaped beam from linear antenna array by finding optimum amplitudes and phases of the array elements using the modified least square method [4]. Chatterjee et al. [5] proposed a technique based on finding optimum phases of the array elements modifying which over existing "zero" phases generates two different patterns. The search has been carried out using Gravitational Search Algorithm (GSA) [5]. In [6], a new technique has been proposed by Chatterjee et al. for obtaining dual beam pair where the states ("on/off") of the array elements are modified using Firefly Algorithm (FA) for generating two different beam pairs. The method greatly simplified the design of the feed network [6].

The paper presents shaped beam synthesis of two ring concentric array of isotropic elements. Two different cases 


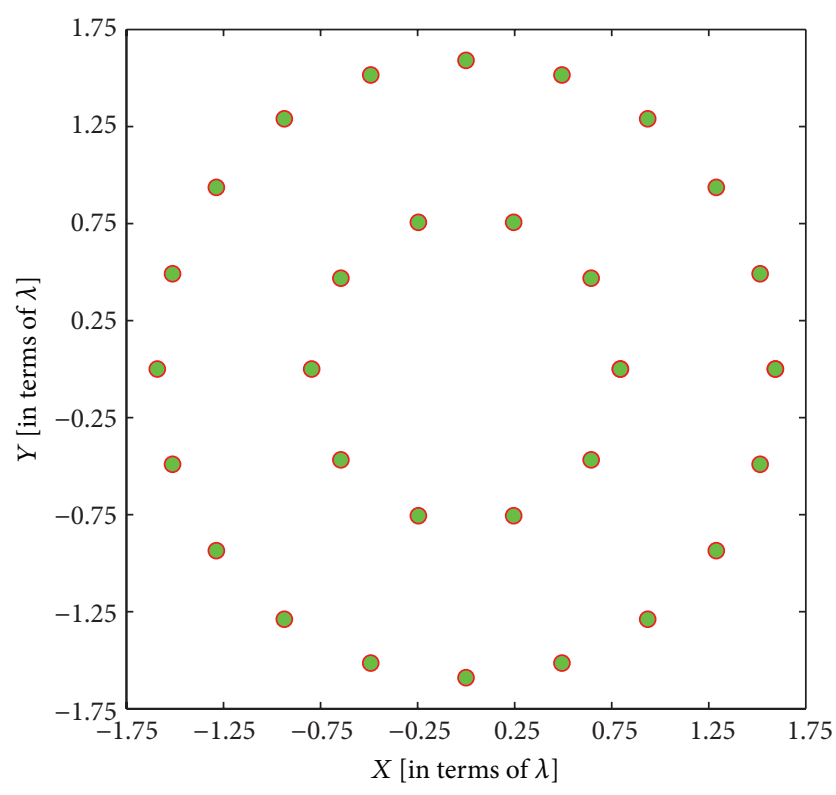

FIGURE 1: Concentric ring array of isotropic antennas in $X-Y$ plane.

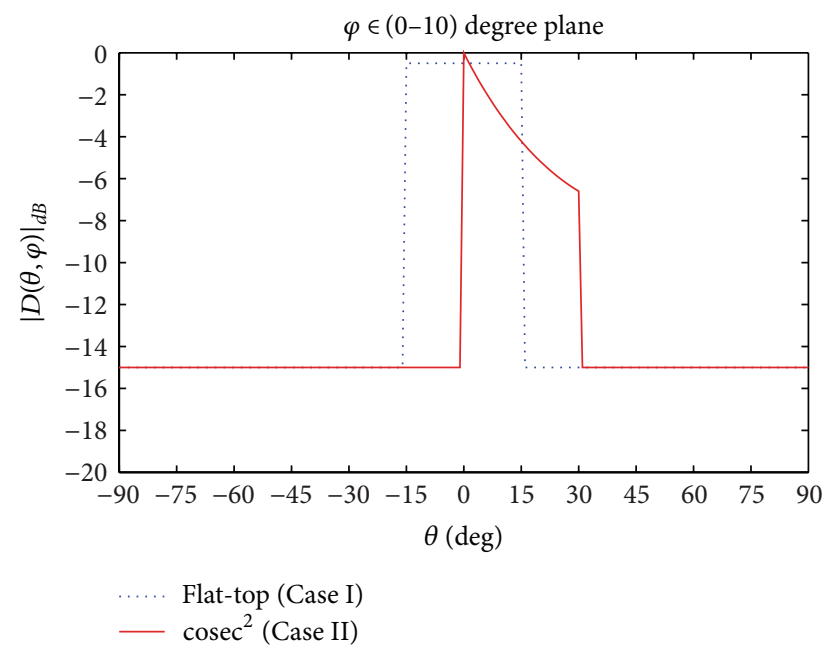

FIGURE 2: Desired patterns under two different design cases.

have been considered. In the first case a flat-top beam is generated from the presented array by finding optimum 4bit amplitudes and 5-bit phases, and in the second case a $\operatorname{cosec}^{2}$ pattern is generated from the same array by finding out another optimum 4-bit amplitudes and 5-bit phases of the elements. In both the cases the optimum discrete excitations are computed in such a manner that the obtained patterns are retaining their desired characteristics within a range of predefined $\varphi$-planes with some minor variations. The optimum discrete amplitudes and phases for the two different cases are computed using Differential Evolution (DE) algorithm [7-11]. To illustrate the effectiveness of DE, the shaped beams under two different cases are computed separately following the above procedure from the same array configuration using Particle Swarm Optimization (PSO)

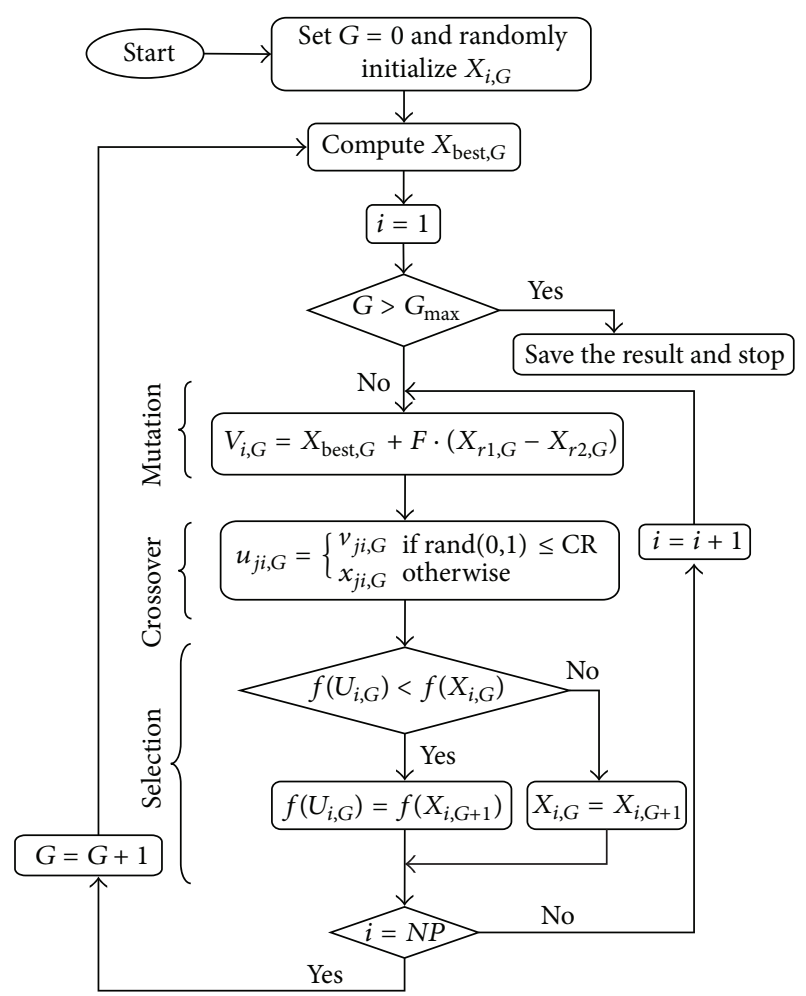

Figure 3: Flow chart of Differential Evolution (DE) algorithm.

$[6,9,12]$ algorithm and Genetic Algorithm (GA) [12-16]. Results clearly show the effectiveness of DE over PSO and GA for both the design cases presented in this problem.

\section{Problem Formulation}

A concentric ring array of isotropic elements is considered. The far field pattern of the array shown in Figure 1 can be written as [6]

$$
A F(\theta, \varphi)=\sum_{m=1}^{M} \sum_{n=1}^{N_{m}} I_{m n} e^{j\left[k r_{m} \sin \theta \cos \left(\varphi-\varphi_{m n}\right)+\alpha_{m n}\right]},
$$

where $M$ is the number of concentric rings; $N_{m}$ is the number of isotropic elements in $m$ th ring; $I_{m n}$ is the excitation amplitude of $m n$th element; $r_{m}$ is the $N_{m} d_{m} / 2 \pi$ radius of the $m$ th ring; $d_{m}$ is the inter element arc spacing of $m$ th circle; $k=2 \pi / \lambda$, represents wave number; $\lambda$ is the wave length; $\theta, \varphi$ is the polar and azimuth angle; $\varphi_{m n}=2 n \pi / N_{m}$ is the angular location of the $m n$th element with $1 \leq n \leq N_{m}$; and $\alpha_{m n}$ is the phase excitation of $m n$th element.

The fitness function for the shaped beam pattern is defined as follows:

$$
F(\rho)=k_{1}\left(\text { peakSLL }^{d}-\max _{\theta \in A}\left\{A F_{d B}^{\rho}(\theta, \varphi)\right\}\right)^{2}+k_{2} \times \Delta,
$$

where $\Delta$ is defined as

$$
\Delta=\sum_{\theta_{\text {ripple }}}\left|A F_{d B}^{\rho}\left(\theta_{\text {ripple }}, \varphi\right)-D\left(\theta_{\text {ripple }}, \varphi\right)_{d B}\right| .
$$

In (2) and (3), $\varphi \in\left(0^{\circ}-10^{\circ}\right)$ plane. 


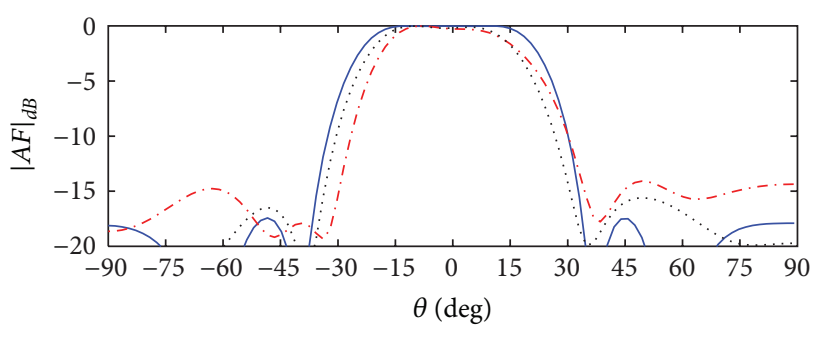

(a)

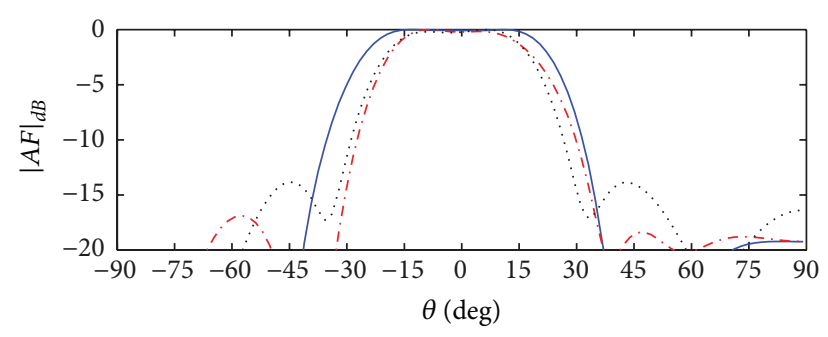

(b)

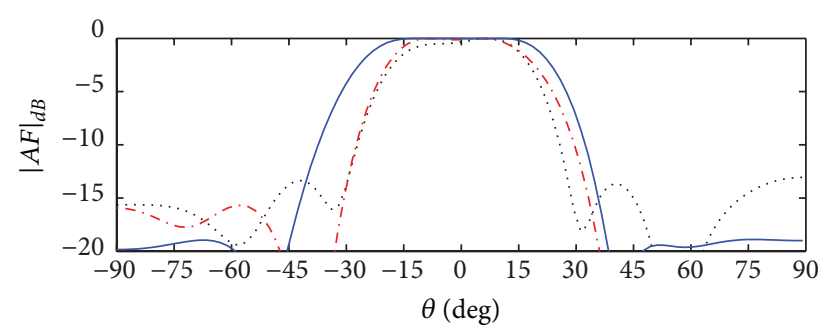

(c)

FIGURE 4: Optimized flat-top patterns from the concentric ring array: (a) for $\varphi=0$ degree plan,e (b) for $\varphi=5$ degree plane, (c) for $\varphi=10$ degree plane.

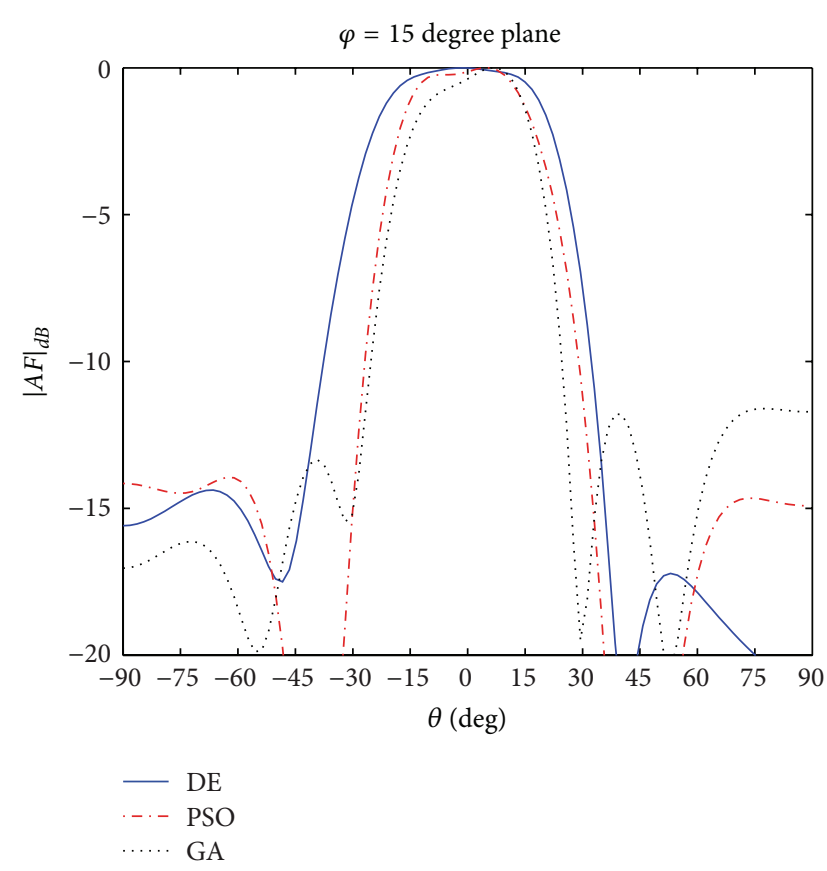

FIGURE 5: Optimized flat-top patterns from the concentric ring array for $\varphi=15$ degree plane.

In (2), $\rho$ is the unknown parameter set responsible for the desired beam pattern for the two different cases. $\rho$ is defined as follows:

$$
\rho=\left\{I_{m n}, \alpha_{m n}\right\}, \quad 1 \leq m \leq M, 1 \leq n \leq N_{m} .
$$

peakSLL ${ }^{d}$ is the desired value of peak SLL for the two different cases. $A$ is the sidelobe region for the shaped beams of flattop and $\operatorname{cosec}^{2}$ patterns. $\theta_{\text {ripple }}$ in (2) represents the range of all " $\theta$ ", for the ripple parameter. The range of $\theta_{\text {ripple }}$ for the flat-top beam is defined as $-15^{\circ}$ to $+15^{\circ}$, and, for the $\operatorname{cosec}^{2}$ beam, the range of $\theta_{\text {ripple }}$ is defined as $0^{\circ}$ to $30^{\circ} . D(\theta, \varphi)_{d B}$ represent desired patterns under two different design cases for $\varphi \in\left(0^{\circ}-10^{\circ}\right)$ plane, shown in Figure 2. $k_{1}$ and $k_{2}$ are the weighting factors to give relative importance in each term of (2). The values of $k_{1}$ and $k_{2}$ are chosen as "one." For synthesis of shaped beam pattern under two different design cases, the fitness function has to be minimized separately under each of the cases by computing optimum set of discrete 4-bit $I_{m n}$ and optimum set of discrete 5-bit $\alpha_{m n}$ among the array elements.

The first part of (2) is proposed to reduce the peak SLL of the optimized beam pattern and second part is proposed to obtain the desired shape of the beam pattern while minimizing the value of $F(\rho)$.

In (2), $\max _{\theta \in A}\left\{A F_{d B}^{\rho}(\theta, \varphi)\right\}$ represents the obtained maximum sidelobe level of the beam pattern within the prespecified range of $\varphi$-plane for the unknown parameter set $\rho$, which has to be determined. The term (peakSLL $\left.{ }^{d}-\max _{\theta \in A}\left\{A F_{d B}^{\rho}(\theta, \varphi)\right\}\right)$ represents the deviation between the desired and obtained values of the peak SLL for the parameter set $\rho$. The second part of (2), which is responsible for obtaining the desired shape of the beam pattern, is defined in (3). In (3), $A F_{d B}^{\rho}\left(\theta_{\text {ripple }}, \varphi\right)$ represents computed values of ripple parameter under different predefined $\varphi$-cut of the beam pattern for the parameter set $\rho$, and $D\left(\theta_{\text {ripple }}, \varphi\right)_{d B}$ are the desired patterns shown in Figure 2. The term $\left|A F_{d B}^{\rho}\left(\theta_{\text {ripple }}, \varphi\right)-D\left(\theta_{\text {ripple }}, \varphi\right)_{d B}\right|$ in (3) represent the deviations between these two parameters and finally their summation is computed to determine the total error in ripple under all predefined $\varphi$-cuts within the entire range of $\theta_{\text {ripple }}$ for the parameter set $\rho$. The minimization of $F(\rho)$ minimizes the two different terms associated with (2), and hence the obtained 


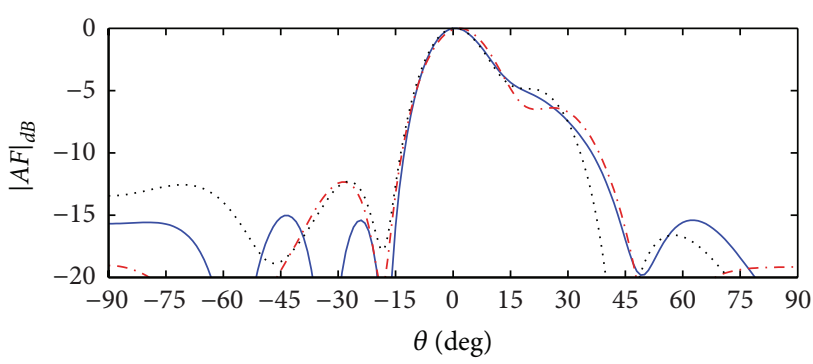

(a)

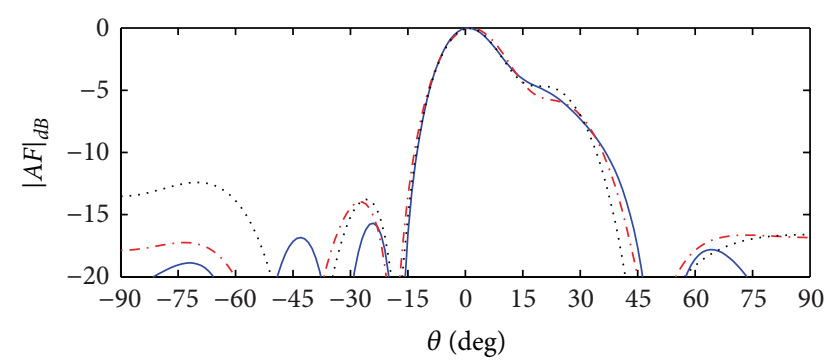

(b)
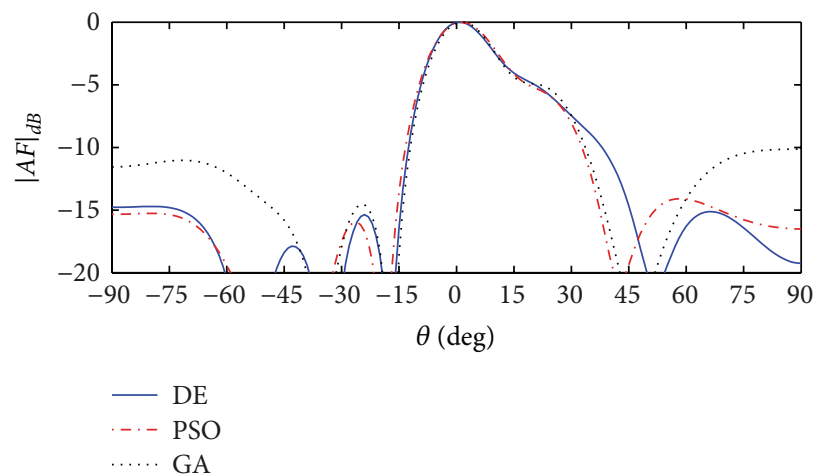

(c)

FIGURE 6: Optimized $\operatorname{cosec}^{2}$ patterns from the concentric ring array: (a) for $\varphi=0$ degree plane, (b) for $\varphi=5$ degree plane, (c) for $\varphi=10$ degree plane.

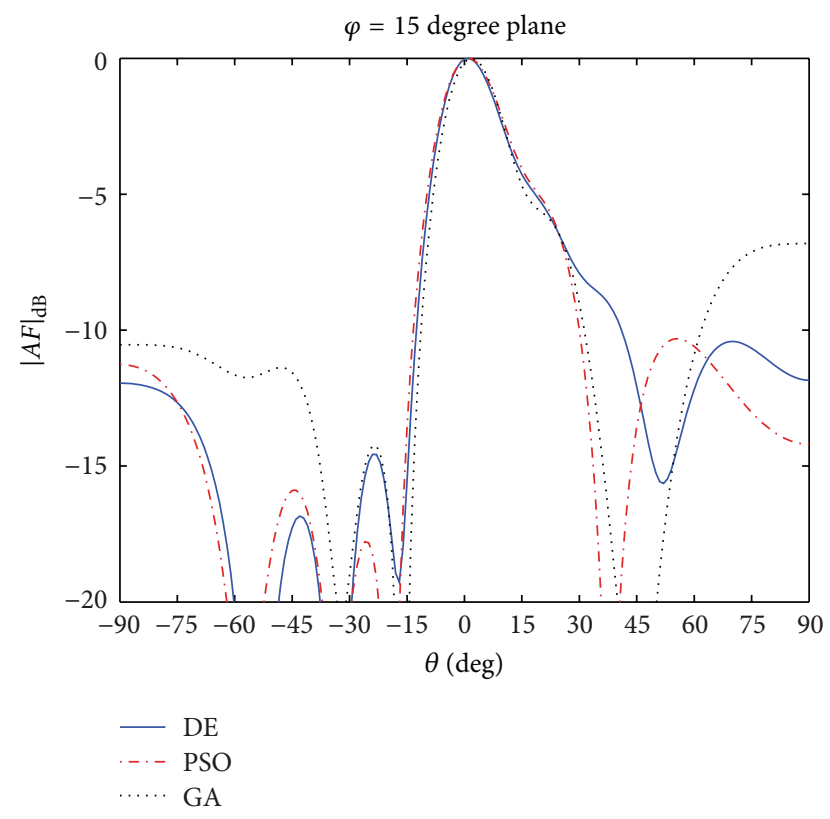

FIGURE 7: Optimized $\operatorname{cosec}^{2}$ patterns from the concentric ring array for $\varphi=15$ degree plane.

beam-pattern approaches towards its desired one for the computed parameter set $\rho$. In this manner, the fitness function of (2) serves the purpose of generating desired shaped beams while minimizing it individually under each of the design cases using DE, PSO, and GA.

\section{Algorithm Overviews and Parametric Setup}

3.1. Overview of Differential Evolution Algorithm. Differential Evolution (DE) algorithm was introduced by Storn and Price. Similar to GA [12-16], DE is also based on population. It is a stochastic optimization method used to minimize an objective function. It has an advantage to find out true global minima and fast convergence using a few control parameters [7-11]. DE algorithm generates a population of $N P$ in $D$ dimensional search space called individuals. The individual of population in generation $G$ can be written as $X_{i, G}=\left\{x_{1 i, G}, x_{2 i, G}, \ldots, x_{D i, G}\right\}, i=1,2, \ldots, N P$. The initial population covered the entire search space. At a generation $G=0$ the initial value of the $j$ th parameter defined as follows: $x_{j i, 0}=\operatorname{rand}(0,1) \cdot\left(x_{j}^{\text {up }}-x_{j}^{\text {low }}\right)+x_{j}^{\text {low }}$, where $i=1,2, \ldots, N P, j=1,2, \ldots, D \cdot \operatorname{rand}(0,1)$ is uniformly distributed random variable within the range $(0,1) ; x_{j}^{\text {low }}$ and $x_{j}^{\text {up }}$ are lower and upper bounds of $j$ th parameter. Three steps mutation, crossover and selection can be described as follows.

Mutation Operation. DE performs mutation operation to generate a mutant vector $V_{i, G}=\left\{v_{1 i, G}, v_{2 i, G}, \ldots, v_{D i, G}\right\}$ for each target vector $X_{i, G}$. In this work, the DE strategy used is "DE/best/1/bin" which is defined as follows [7-10]: $V_{i, G}=$ $X_{\text {best }, G}+F \cdot\left(X_{r_{1}, G}-X_{r_{2}, G}\right) r_{1}, r_{2} \in[1, N P]$ and $r_{1} \neq r_{2} \neq i . F$ is a real and constant factor, satisfying $F \in[0,2]$ and $X_{\text {best, } G}$ is the vector which has best fitness at $G$ th generation.

Crossover Operation. In this operation trial vector $U_{i, G}=$ $\left\{u_{1 i, G}, u_{2 i, G}, \ldots, u_{D i, G}\right\}$ is generated from the target vector $X_{i, G}$ 
TABLE 1: Desired and obtained results for Case I of the design problem.

\begin{tabular}{|c|c|c|c|c|c|}
\hline Specific $\varphi$ cut & \multicolumn{2}{|c|}{ Design parameters } & $\mathrm{DE}$ & PSO & GA \\
\hline \multirow{4}{*}{$\varphi=0$ degree plane } & \multirow{2}{*}{ Peak SLL in $d B$} & Desired & -15.00 & -15.00 & -15.00 \\
\hline & & Obtained & -17.43 & -14.01 & -15.59 \\
\hline & \multirow{2}{*}{ Deviation $(\Delta) d B$} & Desired & 0.00 & 0.00 & 0.00 \\
\hline & & Obtained & 9.60 & 16.33 & 15.11 \\
\hline \multirow{4}{*}{$\varphi=5$ degree plane } & \multirow{2}{*}{ Peak SLL in $d B$} & Desired & -15.00 & -15.00 & -15.00 \\
\hline & & Obtained & -19.22 & -17.08 & -14.54 \\
\hline & \multirow{2}{*}{ Deviation $(\Delta) d B$} & Desired & 0.00 & 0.00 & 0.00 \\
\hline & & Obtained & 9.99 & 14.70 & 15.28 \\
\hline \multirow{4}{*}{$\varphi=10$ degree plane } & \multirow{2}{*}{ Peak SLL in $d B$} & Desired & -15.00 & -15.00 & -15.00 \\
\hline & & Obtained & 18.89 & -16.84 & -13.75 \\
\hline & \multirow{2}{*}{ Deviation $(\Delta) d B$} & Desired & 0.00 & 0.00 & 0.00 \\
\hline & & Obtained & 9.50 & 14.44 & 18.52 \\
\hline
\end{tabular}

and mutant vector $V_{i, G}$. The crossover strategy is defined follows:

$$
u_{j i, G}= \begin{cases}v_{j i, G} & \text { if rand }(0,1) \leq \mathrm{CR} \\ x_{j i, G} & \text { otherwise. }\end{cases}
$$

Crossover factor CR is const in the range of $(1,0)$. The value of $C R$ is taken as 0.2 .

Selection. The operation performs comparison between the objective function values at each trial vector $f\left(U_{i, G}\right)$ and target vector $f\left(X_{i, G}\right)$. The vector which has smaller fitness function value remains in the next generation. Selection operation can be expressed as

$$
X_{i, G+1}= \begin{cases}U_{i, G} & \text { if } f\left(U_{i, G}\right)<f\left(X_{i, G}\right) \\ X_{i, G} & \text { otherwise. }\end{cases}
$$

These three steps are repeated generation by generation until it reaches to its termination condition. Return the best vector in the current population $\left(X_{\text {best }, G}\right)$ as the solution of the optimization problem. The flow chart of Differential Evolution is given in Figure 3.

3.2. Details of Parametric Setup. The individuals of the population for DE, PSO, and GA are considered as

$$
X=\left[\begin{array}{llllllll}
I_{1} & I_{2} & \cdots & I_{K} & \alpha_{1} & \alpha_{2} & \cdots & \alpha_{K}
\end{array}\right] .
$$

The limits of the variables are defined as follows:

$$
\begin{gathered}
0 \leq I_{m} \leq 1, \quad \text { for } m=1,2, \ldots, K \\
-\pi \leq \alpha_{m} \leq \pi
\end{gathered}
$$

The value of $K$ in this problem becomes 30 and the search space dimension becomes 60 .

Based on the guideline provided in [7-11], the population size, scale factor $(F)$, and crossover rate $(C R)$ of DE are chosen as $50,0.8$, and 0.2 . The DE scheme used is: "DE/best/1/bin" and the maximum iteration number is chosen as 3000 .

Swarm size in PSO is taken as 50 and the initial population is chosen randomly. The values of $C 1$ and $C 2$ are chosen as $2[6,9]$. Time-varying inertia weight $(w)$ is considered as decreasing linearly from 0.9 to 0.4 .

The maximum allowable velocity for each of the particle on $d$ th dimension is considered as $0.9 r_{d}[6,9]$, where $r_{d}$ is the difference between the maximum and minimum possible values of decision variables on $d$ th dimension. The termination condition is chosen as a maximum iteration of 3000 .

Population size in GA is taken as 50 and two-point crossover is chosen. Crossover probability and mutation probability are taken as 0.08 and 0.01 , "Roulette Wheel" Selection is considered for the proposed problem, and the termination condition is chosen as a maximum iteration of 3000. Other parametric setups of GA are taken from guidelines given in [12-16].

\section{Simulation Results}

A two ring concentric array of total 30 isotropic elements has been considered. The number of elements in each ring of the array is taken $10 m$, where $m$ is the ring number.

The interelement spacing is considered as $0.5 \lambda$; that is, $d=$ $0.5 \lambda$, and the ring radii are computed as $0.795 \lambda$ and $1.59 \lambda$. The presented results in this section are the best set of results obtained from 20 different runs of each of the algorithm for each individual case.

The design specifications of flat-top beam patterns computed separately using DE, PSO, and GA and their corresponding obtained results in different $\varphi$-planes are shown in Table 1. From Table 1, it can be seen that the obtained values of the design parameters using DE are better than PSO and GA for the flat-top beam pattern.

Three different $\varphi$-cuts of the obtained flat-top beams, computed individually using DE, PSO, and GA are shown in Figure 4. In Figure 4, the presented $\varphi$-cuts are 0 degree, 5 degrees, and 10 degrees. It can be ensured from Figure 4 that the obtained flat-top beams are not restricted in any single $\varphi$-plane within the prespecified range of $0^{\circ} \leq \varphi \leq 10^{\circ}$. To observe the flat-top beam patterns from a different $\varphi$-plane which is outside its prespecified range $\left(0^{\circ} \leq \varphi \leq 10^{\circ}\right)$, a 15 degree $\varphi$-cut of the optimized beam patterns is presented in 


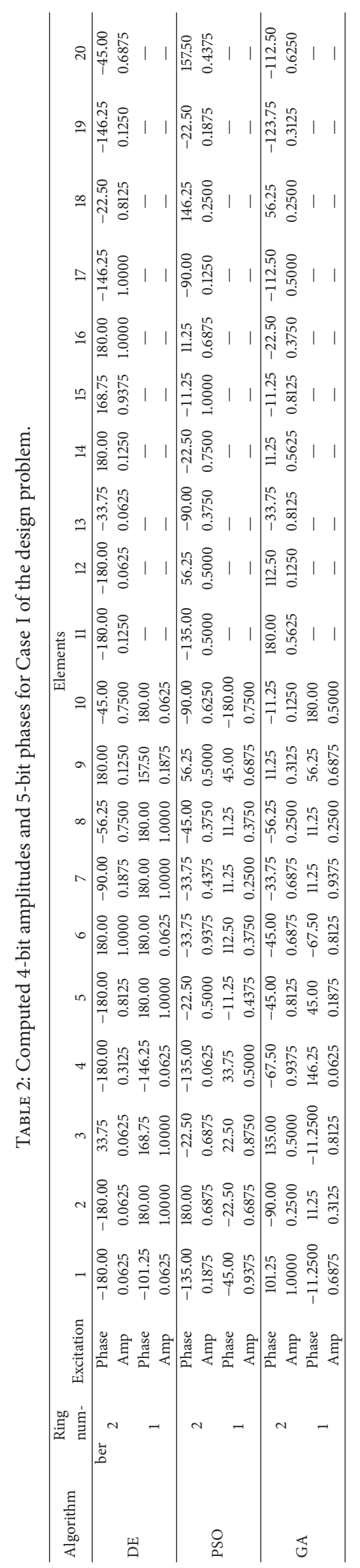


TABLE 3: Desired and obtained results for Case II of the design problem.

\begin{tabular}{|c|c|c|c|c|c|}
\hline Specific $\varphi$ cut & \multicolumn{2}{|c|}{ Design parameters } & $\mathrm{DE}$ & PSO & GA \\
\hline \multirow{4}{*}{$\varphi=0$ degree plane } & \multirow{2}{*}{ Peak SLL in $d B$} & Desired & -15.00 & -15.00 & -15.00 \\
\hline & & Obtained & -15.03 & -12.34 & -12.29 \\
\hline & \multirow{2}{*}{ Deviation $(\Delta)$} & Desired & 0.00 & 0.00 & 0.00 \\
\hline & & Obtained & 11.18 & 22.91 & 13.68 \\
\hline \multirow{4}{*}{$\varphi=5$ degree plane } & \multirow{2}{*}{ Peak SLL in $d B$} & Desired & -15.00 & -15.00 & -15.00 \\
\hline & & Obtained & -15.69 & -13.97 & -12.41 \\
\hline & \multirow{2}{*}{ Deviation $(\Delta)$} & Desired & 0.00 & 0.00 & 0.00 \\
\hline & & Obtained & 12.84 & 15.55 & 15.90 \\
\hline \multirow{4}{*}{$\varphi=10$ degree plane } & \multirow{2}{*}{ Peak SLL in $d B$} & Desired & -15.00 & -15.00 & -15.00 \\
\hline & & Obtained & -14.71 & -14.09 & -10.13 \\
\hline & \multirow{2}{*}{ Deviation $(\Delta)$} & Desired & 0.00 & 0.00 & 0.00 \\
\hline & & Obtained & 13.70 & 16.00 & 15.20 \\
\hline
\end{tabular}

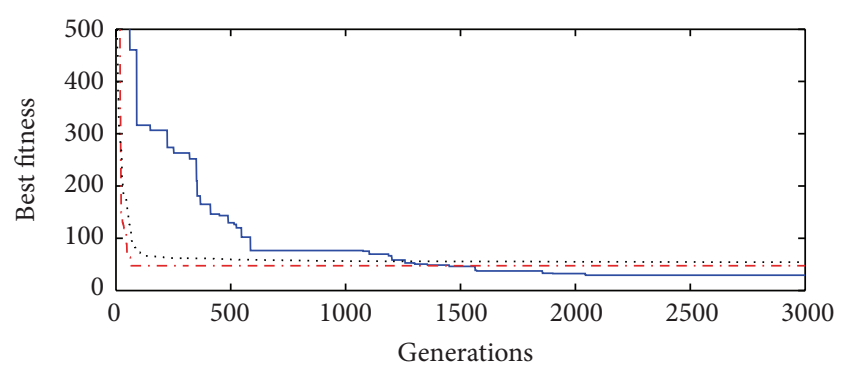

$$
\begin{aligned}
& \text { - DE } \\
& \cdots-\text { PSO } \\
& \text { … GA }
\end{aligned}
$$

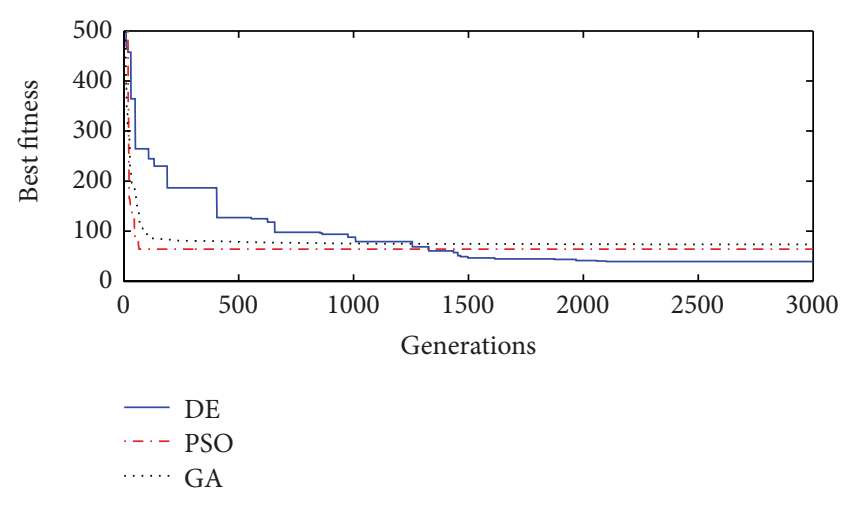

(b)

FIGURE 8: Convergence characteristics of DE, PSO, and GA: (a) for Case I of the design problem (b) and for Case II of the design problem.

Figure 5. From Figure 5, it can be seen that the patterns are deviated from its desired flat-top pattern of Figure 3, because they are not optimized for $\varphi=15$ degree plane. The optimum 4-bit amplitudes and 5-bit phases of the array elements for the flat-top beams computed individually using DE, PSO, and GA are shown in Table 2 .

The design specification for $\operatorname{cosec}^{2}$ beam patterns (Case II) and their corresponding obtained results in three different $\varphi$-planes are shown in Table 3. From Table 3, it can be observed that the performance of DE, in terms of computing the design parameters for $\operatorname{cosec}^{2}$ beam pattern, is better than PSO and GA.

Figure 6 shows three different $\varphi$-cuts of the obtained $\operatorname{cosec}^{2}$ beam patterns, computed individually using DE, PSO, and GA. In Figure 6, the presented $\varphi$-cuts of the optimized $\operatorname{cosec}^{2}$ beam patterns are 0 degree, 5 degrees, and 10 degrees.

Figure 7 shows a 15-degree $\varphi$-cut of the optimized $\operatorname{cosec}^{2}$ beam patterns which clearly shows deviation from its desired $\operatorname{cosec}^{2}$ pattern of Figure 3, because they are not optimized for $\varphi=15$ degree plane. The optimum 4-bit amplitudes and 5-bit phases of the array elements for the $\operatorname{cosec}^{2}$ beams computed individually using DE, PSO, and GA are shown in Table 4.

The comparative performance of DE, PSO, and GA for the two different cases of design problem is shown in Table 5.
Lowest mean fitness value of DE over PSO and GA proves itself best performing algorithm over PSO and GA.

The convergence characteristics of the three algorithms for the two different cases of the presented problem are shown in Figure 8. From Figure 8(a), it can be noticed that the convergence of DE is better than PSO and GA in terms of minimizing the fitness function of (2) for the flattop beam pattern. Similarly, Figure 8(b) clearly shows better convergence of DE over PSO and GA in terms of minimizing the fitness function of (2) for the $\operatorname{cosec}^{2}$ beam pattern.

Table 6 shows the $P$ values obtained through Wilcoxon's rank sum test $[12,17]$ between DE, PSO, and GA for two different cases of design considerations. All the $P$ values are less than 0.05 (5\% significant level) which is a strong proof against null hypothesis indicating that better final fitness value obtained by the best algorithm is statistically significant and has not occurred by chance.

\section{Conclusions}

Synthesis of shaped beam patterns from a concentric ring array antenna using Differential Evolution algorithm has been presented. For synthesis of shaped beam patterns, constrained side lobe and ripple are contemporarily taken into 


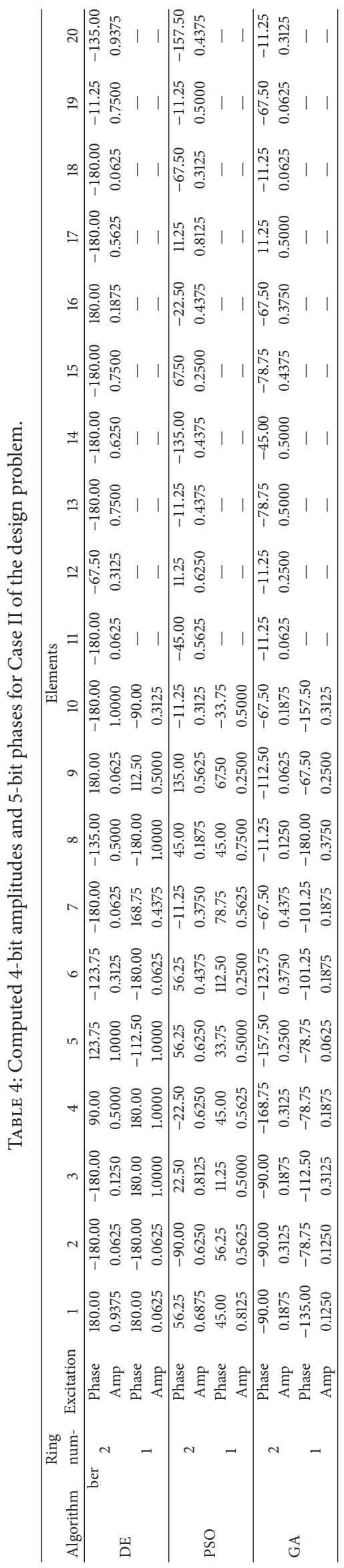


TABLE 5: Comparative performance of DE, PSO, and GA.

\begin{tabular}{|c|c|c|c|c|c|}
\hline Different cases & Algorithm & Best fitness (out of 20) & Worst & Mean & Standard deviation \\
\hline \multirow{3}{*}{ Case I } & $\mathrm{DE}$ & 29.10 & 35.14 & 31.25 & 1.91 \\
\hline & $\mathrm{PSO}$ & 47.47 & 56.96 & 51.33 & 2.89 \\
\hline & GA & 54.25 & 67.73 & 58.27 & 3.89 \\
\hline \multirow{3}{*}{ Case II } & $\mathrm{DE}$ & 39.18 & 45.85 & 42.35 & 1.97 \\
\hline & PSO & 64.03 & 79.90 & 74.40 & 3.49 \\
\hline & GA & 73.67 & 93.87 & 85.69 & 4.32 \\
\hline
\end{tabular}

TABle 6: $P$ values for Wilcoxon's two sided rank sum test.

\begin{tabular}{lcc}
\hline Different cases & Comparison pair & $P$-value \\
\hline \multirow{3}{*}{ Case I } & DE/PSO & $7.0473 e-008$ \\
& DE/GA & $1.0109 e-007$ \\
& $\mathrm{PSO} / \mathrm{GA}$ & $4.1549 e-006$ \\
\multirow{3}{*}{ Case II } & $\mathrm{DE} / \mathrm{PSO}$ & $6.7860 e-008$ \\
& $\mathrm{DE} / \mathrm{GA}$ & $6.7956 e-008$ \\
& $\mathrm{PSO} / \mathrm{GA}$ & $3.9339 e-007$ \\
\hline
\end{tabular}

account by minimizing properly formulated fitness function using Evolutionary Algorithm based procedure. Presented method is capable of producing beam patterns, which retains their desired characteristics within a range of predefined $\varphi$ planes with some minor variations. Results clearly indicate a good agreement between the obtained and desired one.

The presented method incorporates 4-bit amplitudes of the array elements, which ensure that the dynamic range ratio (DRR) remains within the limit of 16 which is helpful for reliable design of the feed network. Discrete excitations also reduced the number of attenuators and the phase shifters and hence are capable of reducing the cost and complexity of the system.

The comparative performance of DE, PSO, and GA clearly shows the superiority of DE over PSO and GA in terms of finding optimum solutions for the presented problem. The quality of the solutions produced individually using DE, PSO, and GA for the two different cases of design considerations is analysed statistically and the superiority of $\mathrm{DE}$ is proven over PSO and GA for the proposed problem.

\section{References}

[1] C. A. Balanis, Antenna Theory, Analysis and Design, John Willy \& Sons, New York, NY, USA, 2nd edition, 1997.

[2] R. S. Elliott, Antenna Theory and Design, John Wiley \& Sons, New York, NY, USA, Revised edition, 2003.

[3] J. A. R. Azevedo, "Shaped beam pattern synthesis with nonuniform sample phases," Progress in Electromagnetics Research B, vol. 5, pp. 77-90, 2008.

[4] J. Lei, G. Fu, L. Yang, and D.-M. Fu, "Wide band linear printed antenna array with low sidelobe cosecant square-shaped beam pattern," Progress in Electromagnetics Research C, vol. 15, pp. 233-241, 2010.

[5] A. Chatterjee, G. K. Mahanti, and P. R. S. Mahapatra, "Design of fully digital controlled reconfigurable dual-beam concentric ring array antenna using gravitational search algorithm," Progress in Electromagnetics Research C, vol. 18, pp. 59-72, 2011.
[6] A. Chatterjee, G. K. Mahanti, and A. Chatterjee, "Design of a fully digital controlled reconfigurable switched beam concentric ring array antenna using firefly and particle swarm optimization algorithm," Progress in Electromagnetics Research B, vol. 36, pp. 113-131, 2012.

[7] R. Storn and K. Price, "Differential evolution: a simple and efficient heuristic for global optimization over continuous spaces," Journal of Global Optimization, vol. 11, no. 4, pp. 341359, 1997.

[8] K. V. Price, R. M. Storn, and J. A. Lampinen, Differential Evolution-A Practical Approach to Global Optimization. Natural Computing, Springer, New York, NY, USA, 2005.

[9] M. A. Panduro, C. A. Brizuela, L. I. Balderas, and D. A. Acosta, "A comparison of genetic algorithms, particle swarm optimization and the differential evolution method for the design of scannable circular antenna arrays," Progress in Electromagnetics Research B, vol. 13, pp. 171-186, 2009.

[10] S. Das, A. Abraham, U. K. Chakraborty, and A. Konar, "Differential evolution using a neighborhood-based mutation operator," IEEE Transactions on Evolutionary Computation, vol. 13, no. 3, pp. 526-553, 2009.

[11] J. Guo and J. Li, "Pattern synthesis of conformal array antenna in the presence of platform using differential evolution algorithm," IEEE Transactions on Antennas and Propagation, vol. 57, no. 9, pp. 2615-2621, 2009.

[12] R. L. Haupt, "Introduction to genetic algorithms for electromagnetics," IEEE Antennas and Propagation Magazine, vol. 37, no. 2, pp. 7-15, 1995.

[13] K. F. Man, K. S. Tang, and S. Kwong, "Genetic algorithms: concepts and applications," IEEE Transactions on Industrial Electronics, vol. 43, no. 5, pp. 519-534, 1996.

[14] J. M. Johnson and Y. Rahmat-Samii, "Genetic algorithms in engineering electromagnetics," IEEE Antennas and Propagation Magazine, vol. 39, no. 4, pp. 7-21, 1997.

[15] D. Marcano and F. Durán, "Synthesis of antenna arrays using genetic algorithms," IEEE Antennas and Propagation Magazine, vol. 42, no. 3, pp. 12-20, 2000.

[16] M. A. Panduro, A. L. Mendez, R. Dominguez, and G. Romero, "Design of non-uniform circular antenna arrays for side lobe reduction using the method of genetic algorithms," International Journal of Electronics and Communications, vol. 60, no. 10, pp. 713-717, 2006.

[17] M. Hollander and D. A. Wolfe, Nonparametric Statistical Methods, John Wiley \& Sons, New York, NY, USA, 2nd edition, 1999. 

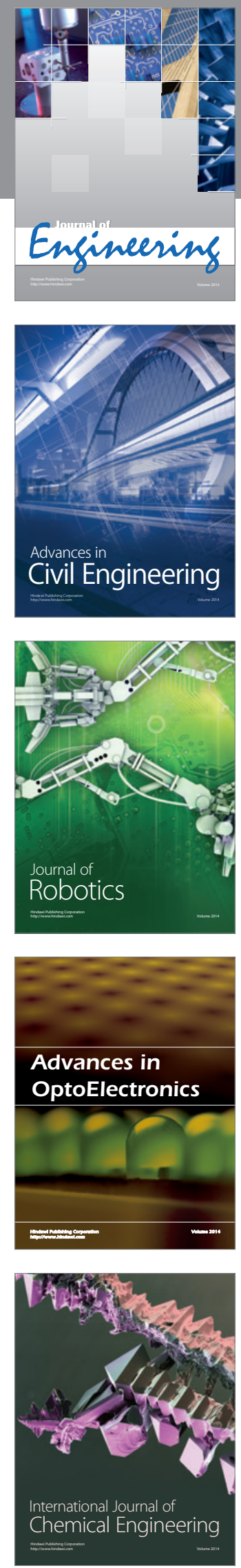

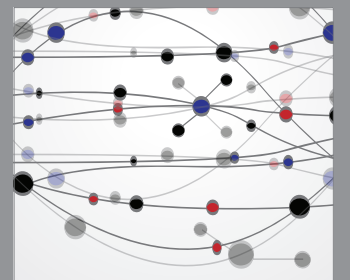

The Scientific World Journal
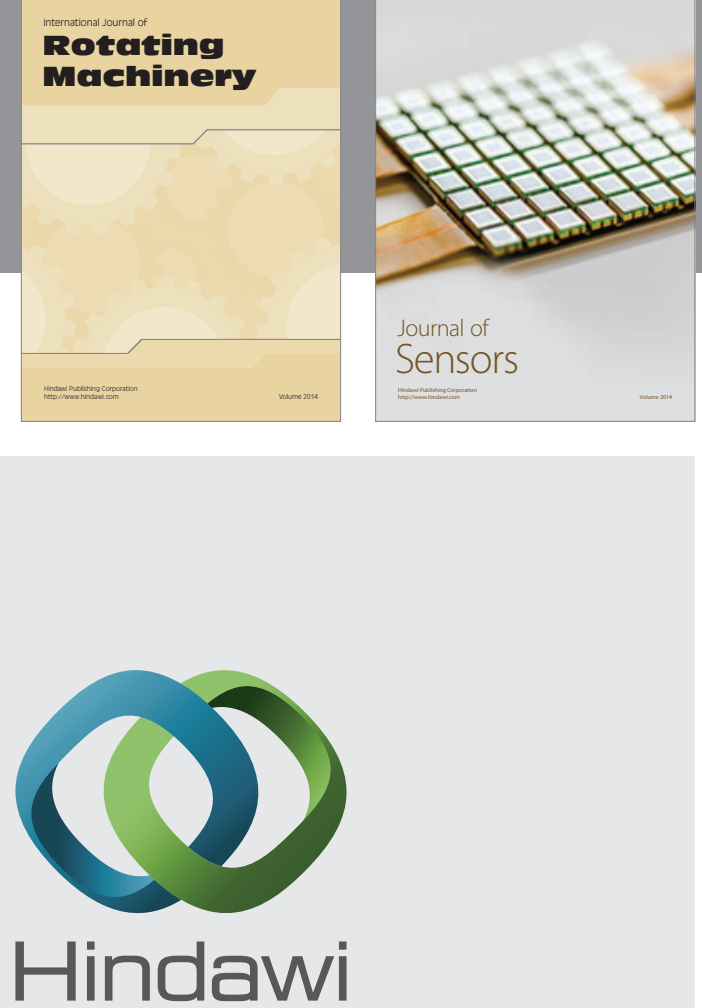

Submit your manuscripts at http://www.hindawi.com
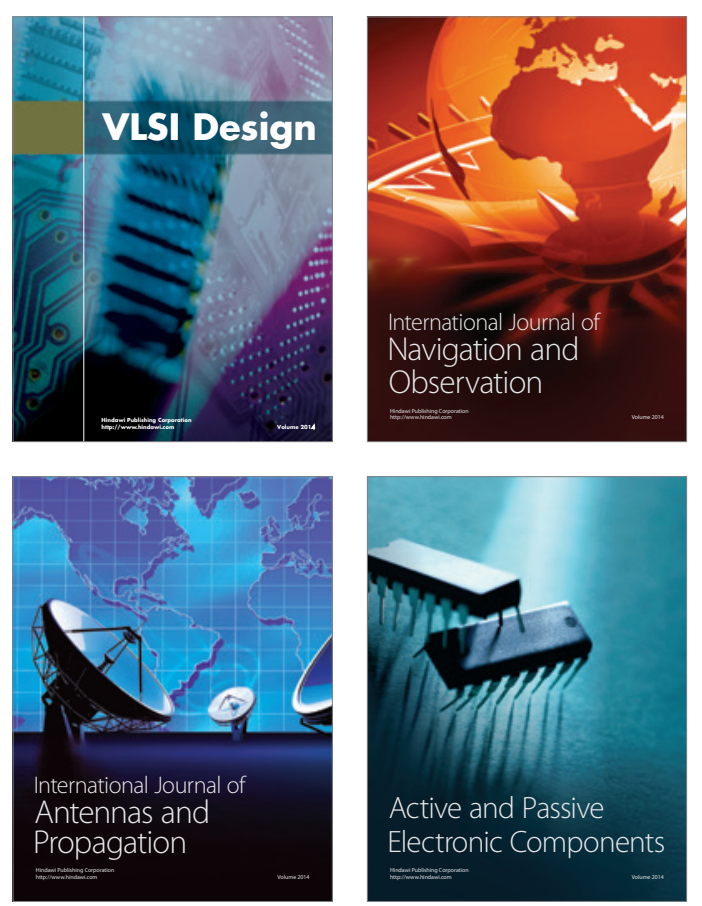
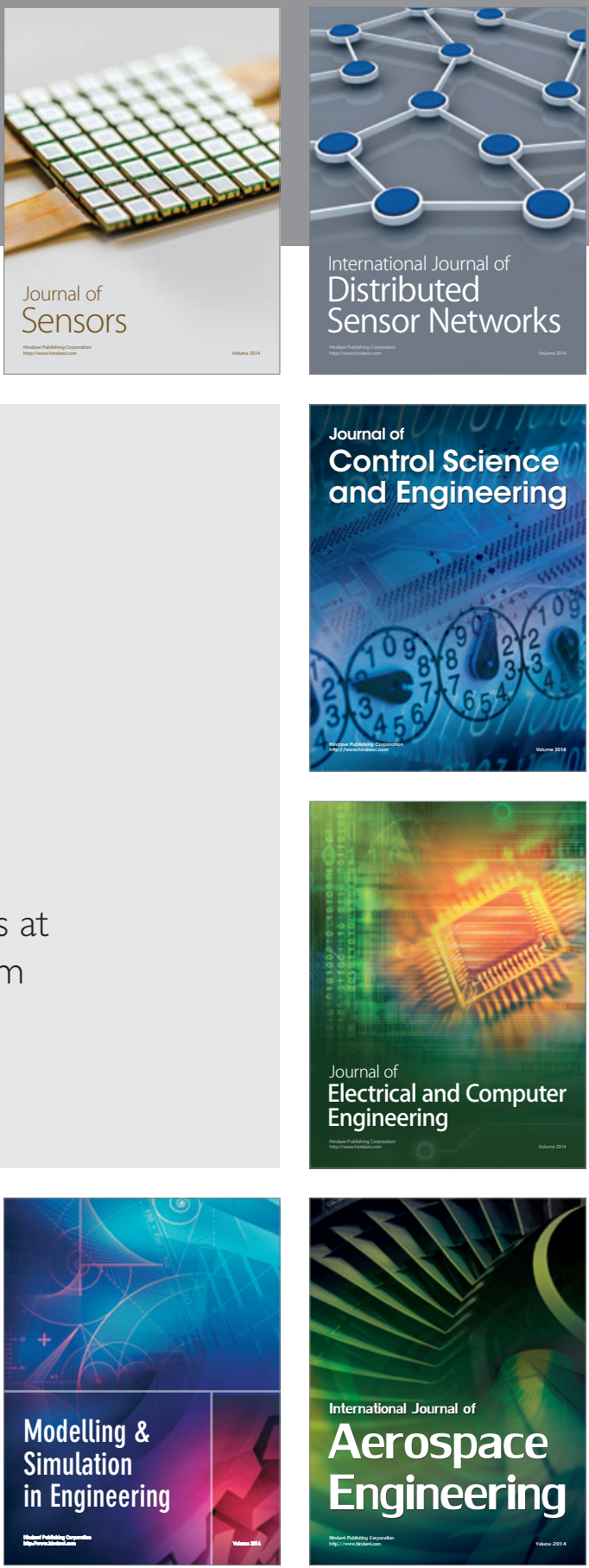

Journal of

Control Science

and Engineering
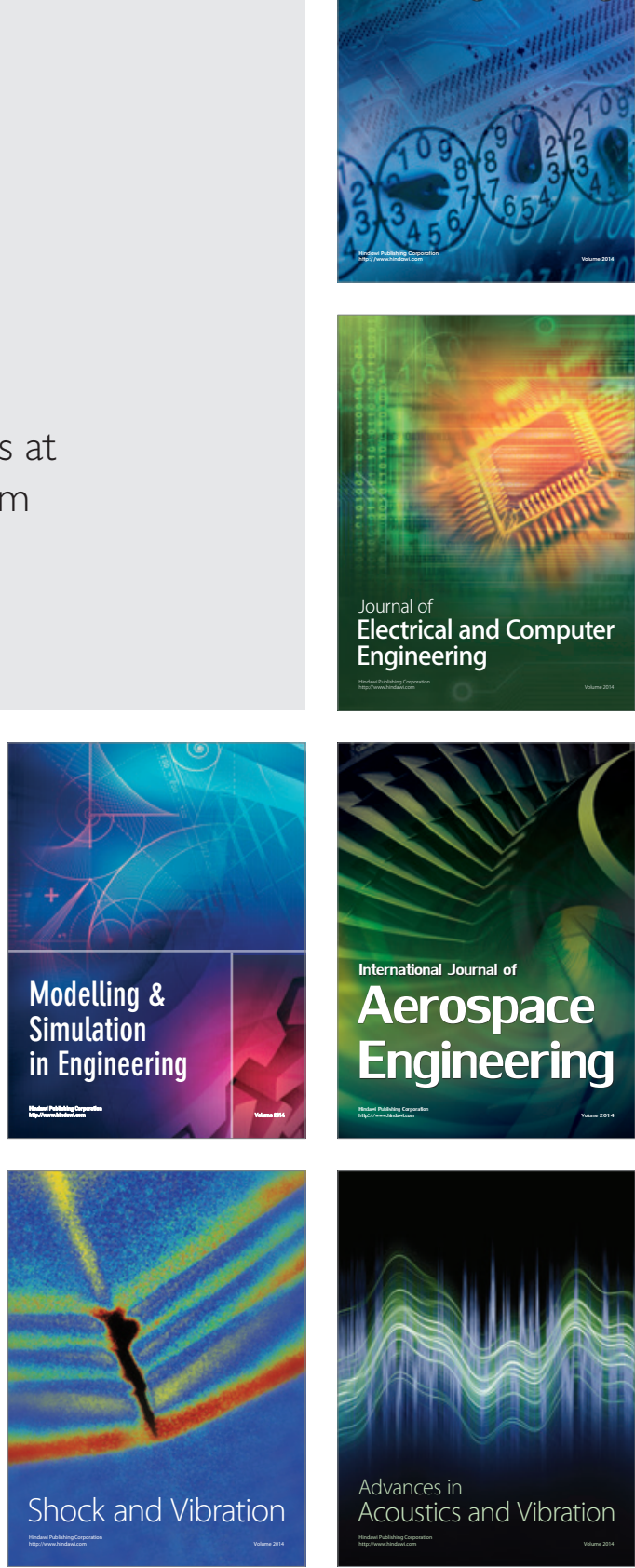